International Journal of Wireless \& Mobile Networks (IJWMN) Vol. 4, No. 2, April 2012

\title{
EFFECTS OF NOVEL LONG LIFE ROUTING ALGORITHMS ON IEEE 802.16 THROUGHPUT IMPROVEMENT METHODS FOR VARIOUS VEHICULAR VELOCITIES
}

\author{
Barbaros Preveze \\ Department of Electronics and Communication Engineering, Çankaya University, \\ Ankara, Turkey \\ b.preveze@cankaya.edu.tr
}

\begin{abstract}
In this paper, effects of Fastest Path Routing, Ant-Colony Routing, Associativity Based Routing (ABR) and other novel proposed Associativity Based Long Life Routing algorithms, on novel proposed throughput improvement algorithm Most Congested Access First (MCAF), minimizing the packet loss ratio and improving the throughput of a multi-hop mobile WIMAX network are investigated for various vehicular velocities. The proposed Long Life Routing algorithms are shown to provide more throughput improvement for all vehicular velocities and provide longer life routes.
\end{abstract}

\section{KEYWORDS}

Throughput, Routing, 802.16j, Multimedia

\section{INTRODUCTION}

The idea of Cognitive Radio was first presented officially by Joseph Mitola III in a seminar at KTH, The Royal Institute of Technology, in 1998. Then an article is published [1] by Mitola and Gerald Q. Maguire, Jr one year later. Mitola introduced this research as a baby step in a potentially interesting research approach [1] and described Cognitivity later as; "The point in which wireless personal digital assistants (PDAs) and the related networks are sufficiently computationally intelligent about radio resources and related computer-to-computer communications to detect user communications needs as a function of use context, and to provide radio resources and wireless services most appropriate to those needs" [2]. By the appearance of this novel concept new challenges in the literature to the resource allocation and design of WIMAX relay-based system have been introduced. Most of the works in the literature attempt to improve the system throughput by trying to cooperate the primary (licensed) users and secondary (unlicensed) users for efficient resource allocation [3, 4]. However, there are only little research works specifically on multi-hop 802.16j networks at present [5] that try to improve the throughput with design of WIMAX relay based system.

In [3], the authors propose a novel method for flexible channel cooperation which allows secondary users to freely optimize the use of channels for transmitting primary data along with their own data in order to maximize the throughput performance.

DOI : $10.5121 /$ ijwmn.2012.4206 
International Journal of Wireless \& Mobile Networks (IJWMN) Vol. 4, No. 2, April 2012

In [4], the throughput potential of cognitive communication is explored considering opportunistic communication as a base line and the throughput improvements are offered by the overlay methods. The authors focus on determining the throughput potential of Cognitive Radio for various transmission powers of the secondary nodes and determining the optimal amount of licensing. However design of WIMAX relay-based system, effects of routing methods and effects of vehicular velocities are not considered in [3] and [4].

In [5], a study of the performance of transparent mode relay-based 802.16j systems is described and design of WIMAX relay-based system is considered. However effects of routing algorithms and effects of vehicular velocities are not considered in this work and only 5\% throughput improvement is provided with almost twice signaling overhead where we have provided the throughput improvement for different number of nodes $(\mathrm{N})$ in a range between $15 \%$ (for $\mathrm{N}=24$ ) and 36\% (for $\mathrm{N}=4$ ) as seen in Fig.5. According to authors [5], the work already demonstrates the necessity of finding other ways to improve $802.16 \mathrm{j}$ network throughput performance.

In [6], the authors address the problem of assigning channels to Cognitive Radio transmissions assuming one transceiver per Cognitive Radio in order to maximize the number of simultaneous Cognitive Radio transmissions with respect to spectrum assignment. They provide 50\% improvements on the network throughput for single hop scenarios by decreasing the blocking rates of Cognitive Radio transmissions. However effects of routing algorithms and effects of vehicular velocities are not considered in this work and only $20 \%$ improvement is provided for multi-hop scenarios, where we have provided the throughput improvements up to $36 \%$ (for $\mathrm{N}=4$ ) as seen in Fig.5.

In our system we use the proposed OFDMCAF method which is a combination of OFDMA and TDMA [7, 8, 9]. It uses additional novel throughput improvement methods to decrease the packet loss ratio and improve the throughput of a unicast network.

In [10], simulation throughput results of each throughput improvement method are focused on, only for a fixed number of nodes $(\mathrm{N}=6)$. In [11], the simulation results are generalized and evaluated for varied number of nodes $(\mathrm{N})$ and it is shown that the simulation results evaluated for pure network match with the results of another work [12] in the literature. In [11] the probabilistic calculation of packet loss rates of each method is also formulated and the evaluated packet loss rate calculation results are confirmed by the evaluated simulation results. The calculation of the bandwidth wastage appeared in [11] for the first time. However, in this work, the results evaluated by 3 different works in the literature $[12,13,14]$ are used to confirm the results of the simulations and the calculations we have evaluated. The throughput calculation with/without bandwidth wastage is formulated and generalized for different $\mathrm{N}$ values using the changes in; number of nodes, average hop counts, packet loss rates and number of successfully sent/lost video/voice/data packets. Then the results of the calculations evaluated for pure network are confirmed by the simulation results of same cases for each $\mathrm{N}$ value. It is shown that the real time packet transmissions have been provided with full success, the packet loss rate of non-real time data packets has been minimized and the throughput has been improved by each novel method. Finally, as the main purpose of this paper, the effects of Fastest Path, Ant-Colony, Associativity Based Routing (ABR) and novel proposed Long life routing algorithms on novel throughput improvement methods are investigated. It is shown in this paper that, even all routing algorithms improve the system throughput; proposed ABR methods provide greater throughput improvement for all proposed throughput improvement methods for all vehicular velocities. But, the amounts of improvement decreases by increase of vehicular velocity for all cases. 


\section{THROUGHPUT OF MOBILE AD-HOC NETWORKS}

In order to confirm the correctness of our simulation results, the pure system simulation results are compared with the results of the calculations or simulations ones evaluated in the literature $[12,13,14]$ using the same parameter sets of [12],[14] and our simulation system. The resimulated results of the works $[12,13,14]$ for the parameters used our simulation are shown in Fig.1. Note that the results of [12] are evaluated using (1) [12], results of [13] are evaluated using (2) [13] and the results of [14] are evaluated using (3) [14]. Our results are consistent with $[12,13,14]$.

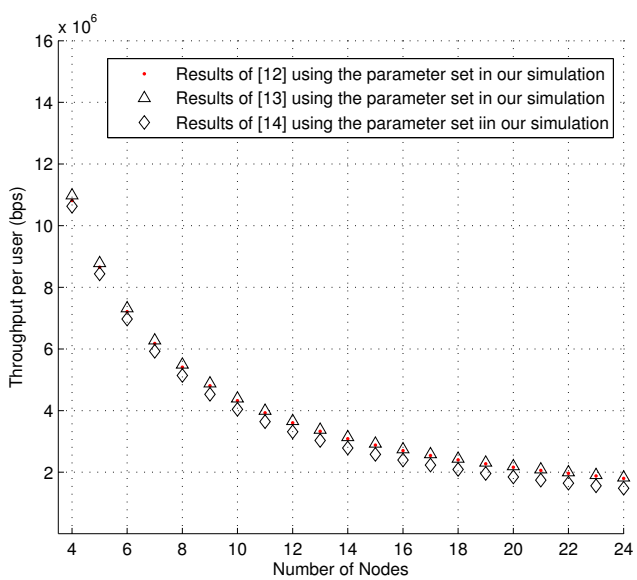

Fig. 1. Comparative analysis for throughput results of different works

\subsection{Asymptotic throughput analysis of unicast transmission}

One of the resultant formulations of the works in the literature that evaluates the throughput of a unicast system by asymptotic analysis is given in (1) [12]. It is also divided by the number of nodes to evaluate the throughput per user.

$$
\begin{aligned}
& \mathrm{Ru} \square B \log _{2}\left(1+\frac{\rho_{0} \ln (N)}{d_{c}{ }^{n}}\right)+\frac{B n}{2 \ln (2)} \\
& \text { where } \rho_{0}=\frac{P}{N_{0}} \times K \times \beta \\
& E\left\{R_{i}^{s}\right\}=\frac{1}{N} \times E\left\{R_{\text {total }}^{\text {Hom }}\right\} \\
& =\frac{1}{N} \times B \times \log _{2}\left(1+\beta \times E\left\{\Gamma_{\text {eff }}\right\}\right)
\end{aligned}
$$


On the other hand, there is another asymptotic analysis done [13] that the throughput per user is formulated there as given by (2). " $\Gamma_{\text {eff }}$ " in (2) is the average effective SNR. The results illustrated in Fig. 1 are evaluated for all works using the same parameter values as in our simulation program $\left(\mathrm{B}=10 \mathrm{MHz} ; \mathrm{FL}=5 \mathrm{~ms} ; \mathrm{SNR}=103 ; \mathrm{dc}=50 \mathrm{~m} ; \mathrm{n}=2(\right.$ freespace $\left.) ; \mathrm{K}=(0 \mathrm{~dB}) ;{ }_{-}=0: 02\right)$. In order to confirm the correctness of our pure system simulation and calculation results, the results of [12] in Fig.1 which exactly match with the results of [13, 14] are carried on Fig.5 with the legend "Unicast analysis results of pure OFDMA without Adaptive Rate (AR), Buffer management (BM) and Spectral Aids (SA)". It's shown in Fig.5 that," simulation and calculation results of pure OFDMCAF without AR, BM and SA", match with "Unicast analysis results of pure OFDMA without Adaptive Rate (AR), Buffer management (BM) and Spectral Aids (SA)" especially for large number of nodes as depicted in [12]. It is also shown that, "Calculation results of pure OFDMA system without AR, BM and SA" match exactly with "Unicast analysis results of pure OFDMA without Adaptive Rate (AR), Buffer management (BM) and Spectral Aids (SA)".

\subsection{WIMAX throughput evaluation of conventional relaying}

The throughput evaluation of conventional relaying is also evaluated using (3) [14], with the case we have in our simulation that relay stations are in use and one can transmit at once.

$$
B R=\frac{R_{-} O F D M(R S)}{\operatorname{LoF}\left(\sum_{i=1}^{n} \frac{S S G 1_{i}}{b p s_{i}}+\sum_{i=1}^{n} \frac{S S G 2_{i}}{b p s_{i}}+\frac{1}{b p s} \sum_{i=1}^{n} S S G 2_{i}\right)}
$$

\section{SIMULATION PROGRAM}

We have developed an event-driven simulation program using MATLAB, in which the movements, locations and buffer states of the nodes, the organization of the packets in the buffer of each node, selected routes for each packet, instant data generation/transmission rates, and instant overall throughput values are all observable from the screen during the simulation. In our simulation, $\mathrm{N}$ independent and uniformly distributed mobile relay nodes are considered to communicate with each other in a cell structure. The overall algorithm that the nodes use in the simulation is given in Fig. 2 where any simulation parameter value can also be changed to any desired value. 
International Journal of Wireless \& Mobile Networks (IJWMN) Vol. 4, No. 2, April 2012

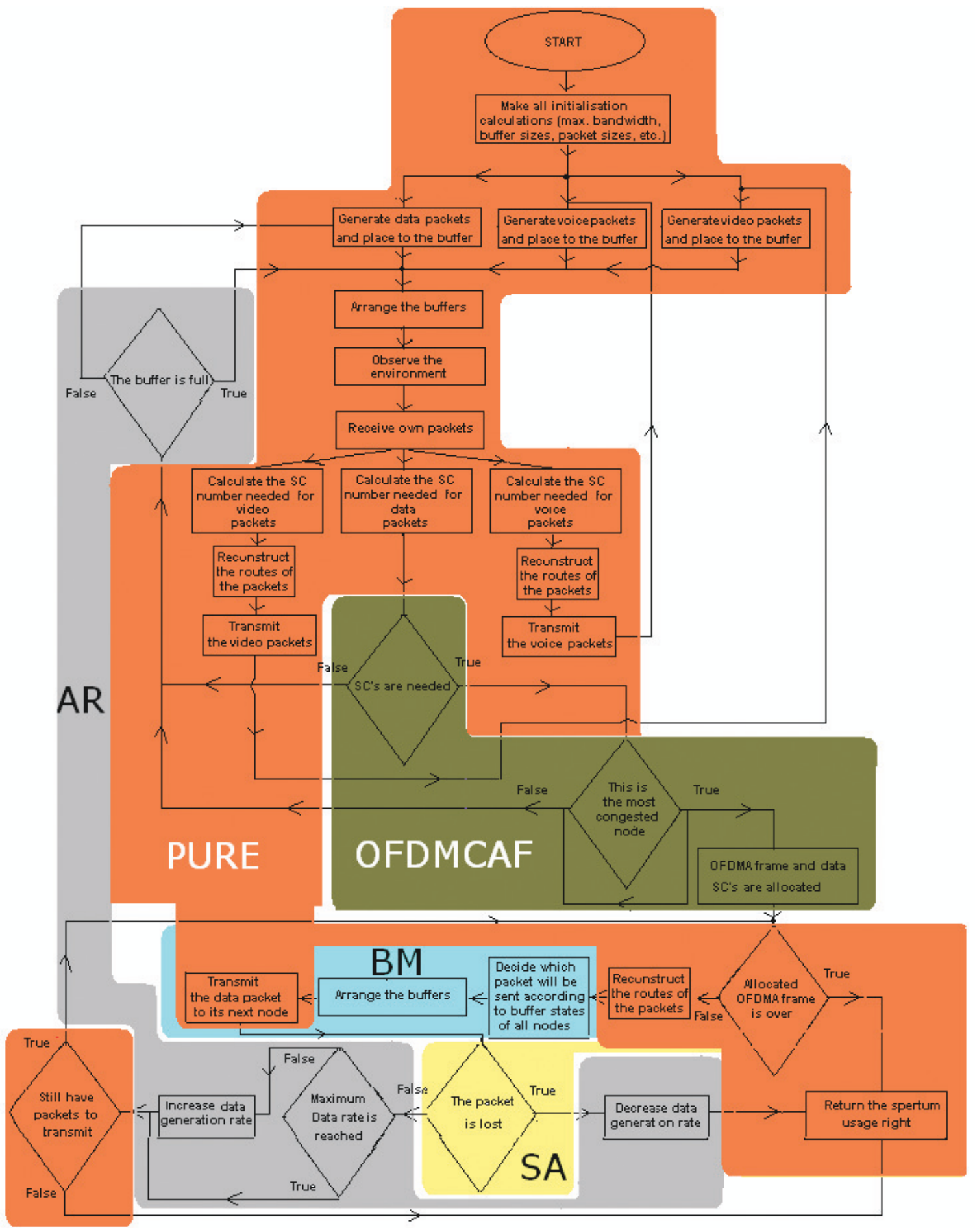

Fig. 2. The overall algorithm used by each node in the simulation in case of using OFDMCAF with AR, BM and SA It is also assumed for best effort that we have unlimited packet generation at data queues of the nodes for which the transmission rate changes according to network conditions. 
International Journal of Wireless \& Mobile Networks (IJWMN) Vol. 4, No. 2, April 2012

\subsection{Parameters used in the simulation and determination of the maximum spectral usage}

In the simulation program the maximum spectral usage that a system may provide is determined using the given input parameters between (4) - (10).

$$
\begin{gathered}
\text { Modulation }(\mathrm{QAM})=64 \\
\text { Frame Length }(F L)=5 \mathrm{~ms} \\
\text { Forward Error Correction }(F E C)=3 / 4 \\
\text { Sub-channel Capacity }(\mathrm{SCC})=0.95 \mathrm{Mbps} \\
\text { Data symbols per Frame }(D S P F)=44 \text { of } 48 \\
\text { Number of sub-channels }(N O S)=30 \\
\text { Bandwidth }(B)=10 \mathrm{MHz} \\
\text { Frames per second }(F P S)=1 / F L f / s \\
\text { Bits per symbol }(\text { BPS })=\log _{2}(Q A M) \times N O D S \mathrm{~b} / \mathrm{sym} .
\end{gathered}
$$

where the abbreviation NODS in (12), expresses the number of data sub-carriers.

$$
\begin{gathered}
\text { Bits per symbol with FEC }(B P S W F)=F E C \times B P S \text { b / sym } \\
\text { Minimum Allocatable Unit }(M A U)=B P S W F / N O S \text { bits } \\
\text { sub - channel data rate in a frame with FEC }(S C D R F W F)=D S P F \times M A U b / f \\
\text { Symbol rate per sec. }(S R P S)=F P S \times D S P F \text { sym. } / \mathrm{s}
\end{gathered}
$$

Using the size of Data Packet (DTPS) as 150 Bytes, Voice Packet size (VCPS) as 10 Bytes [7], Initial Reference Video Packet size (VDPSref) as 512 Bytes, and using other parameters as defined by the standards [7,9] given in (4) - (10) the parameters are calculated using the equations in (11)- (16);

from (12), we have;

$$
\begin{aligned}
\text { Bits per symbol }(\text { BPS }) & =6 \text { bits } / \text { subcarrier } \times 720 \text { subcarrier } \\
& =4320 \mathrm{~b} / \mathrm{sym}
\end{aligned}
$$


from eq. 13, we have;

$$
\begin{aligned}
\text { Bits per symbol with FEC }(\text { BPSWF }) & =\frac{3}{4} \times 4320 \\
& =3240 \mathrm{~b} / \mathrm{sym} .
\end{aligned}
$$

For $\operatorname{NOS}=30, \mathrm{~B}=10 \mathrm{MHz}$, using eq. 13, eq. 14 and the result of eq. 18 we have;

$$
\begin{aligned}
\text { Minimum Allocatable Unit }(\text { MAU }) & =3240 / 30 \\
& =108 \text { bits }
\end{aligned}
$$

Considering a device is allotted one sub-channel (SC) in every frame, there will be 200 frames/sec (from eq. 11) with DSPF= 44 OFDM data symbols/frame each, so the symbol rate results with 8800 using eq. 16 as in eq. 20 .

$$
\begin{aligned}
\text { Symbol rate per sec. }(\text { SRPS }) & =(200 \times 44) \\
& =8800 \text { data sym. } / \mathrm{s} .
\end{aligned}
$$

As the resultant value is also given in eq. 7, the value of sub-channel capacity with FEC can be calculated [7] by eq. 21 ,

$$
\begin{aligned}
\text { Sub }- \text { channel capacity with FEC }(\text { SCC })=(\text { MAU } & \times \text { SRPS })) \\
& =108 \mathrm{bits} \times 8800 \mathrm{sym} . / \mathrm{s} . \\
& =950400 \mathrm{bps} .
\end{aligned}
$$

Then, the maximum spectrum usage (MSU) can be calculated as in eq. 22, by multiplying the sub-channel capacity with the number of sub-channels.

$$
\begin{aligned}
\text { Max. spectrum usage }(M S U) & =S C C \times N O S \\
& =950400 \mathrm{bps} \times 30 \\
& =28.512 \times 10^{6} \mathrm{bps} \\
& =3564000 \mathrm{Bps}
\end{aligned}
$$

The calculated result of eq. 22 also matches with the other results evaluated in the literature [7, 9].

\subsection{Determination of maximum spectral usage with/without bandwidth wastage}

In $[10,11]$ the bandwidth wastage of voice packets in a second (Wastage_voice) for a given $\mathrm{N}$ value is calculated by (23).

$$
\text { Wastage }_{N_{\text {voice }}}=\left(\left(N \times \frac{M S U}{N O S \times \frac{1}{F L}}\right)-\left(V C P S \times(N+(N-1) \times N) \times V P S R_{\text {voice }}\right)\right) \times \frac{1}{F L} B p s
$$


Then the maximum possible successful spectral usage ( MSU) taking account the bandwidth wastage is calculated as in (24) by using the given parameters and by subtracting the total bandwidth wastage amount calculated in (23) from maximum spectral usage.

$$
M S U_{\text {with_wastage }}=M S U-\text { Wastage }_{N_{\text {voice }}} \text { Bps }
$$

The provided throughput amount not taking account (without) the bandwidth wastage is also calculated in $[10,11]$ as given in $(25)$ where AHC expresses the average hop count.

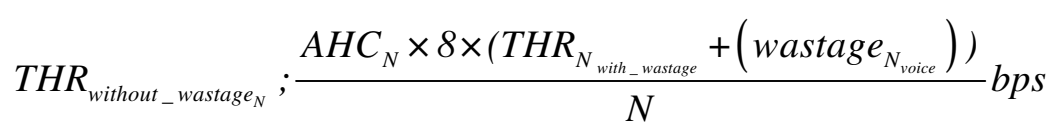

By a simple check for $\mathrm{N}=6$ and for "no method is active" case, using the average hop count (AHC) retrieved from the fastest path routing simulations for $\mathrm{N}=6$ in the given area $(\mathrm{AHC}=3)$, we have the resultant throughput per user without bandwidth wastage as in (26) where bandwidth wastage amount is calculated as 640800 Bytes from (23) and applied to (25) (MSU = 3564000 Bytes; NOS = $30 \mathrm{SC} ; \mathrm{FL}=5 \mathrm{~ms} ; \mathrm{V}$ CPS $=10$ Bytes; VPSR $_{\text {voice }}=1$ ).

$$
\begin{aligned}
T_{H} R_{\text {without_wastage }_{N}} & =\frac{3 \times 8 \times(1225488+(640800))}{6} \mathrm{bps} \\
& =7465152 \mathrm{bps}
\end{aligned}
$$

\subsection{Calculation of the packet sizes of multi-hop mobile WIMAX}

\subsubsection{Calculation of real time multimedia packet sizes}

The nodes can use any voice/video packet sending rates (VPSR voicelvideo $)$ and the video packet size (VDPS) is calculated to be equal to capacity of a single sub-channel in a frame by dividing the maximum spectral usage to the number of sub-channels and number of frames in a second. Since we use the $\mathrm{FL}=5 \mathrm{~ms}$ and $\mathrm{NOS}=30$ in our system, the video packet size can be calculated as in (27). According to [7], $16 \mathrm{kbps}$ voice packets can be considered due to low latency requirements and the voice packet size is calculated as in (28), where our system has 200 frames in a second with frame length of $5 \mathrm{~ms}$.

$$
\begin{gathered}
\text { VDPS }= \\
=\frac{\frac{M S U}{N O S}}{\frac{1}{F L}}=\frac{\frac{3564000 \text { bytes } / \mathrm{s}}{30 \mathrm{SC}}}{200 \mathrm{fps}}=594 \mathrm{Bps} \\
V C P S=\frac{16 \mathrm{kbits}}{200}=10 \text { Bytes }
\end{gathered}
$$




\subsubsection{Calculation of non-real time data packet sizes}

Number of sub-channels in a frame not used by video/voice packets and allocated for data transmission can be calculated as in (29) where TSCFDT expresses the total number of sub-channels allocated for data packets, TSCFVD expresses the total number of sub-channels allocated for video packets and TSCFVC expresses the total number of sub-channels allocated for voice packets.

$$
T S C F D T=(N O S-(T S C F V D+T S C F V C)
$$

The chosen data packet size (DTPS) closest to initially set one $\left(D T P S_{\text {ref }}\right)$ is calculated as in (30) such that multiple of the resultant packet size exactly fit to the sub-channel without any bandwidth wastage where sub-channel data rate in a frame with FEC (SCDRFWF) is calculated as in (27). Note that for $V D P S_{\text {ref }}=512$ Bytes, we have the resultant video packet size (VDPS) as 594 Bytes using (30) for no bandwidth wastage by video packets.

$$
\begin{aligned}
\text { DTPS } & =\frac{(S C D R F W F)}{\text { floor }\left(\frac{S C D R F W F}{D T P S_{\text {ref }}}\right)} \\
& =\frac{594}{\text { floor }\left(\frac{594}{150}\right)}=198 \text { Bytes }
\end{aligned}
$$

\subsection{Calculation of the buffer sizes}

The calculated data buffer size (CDBS) for data and video/voice buffer sizes evaluated for our system are formulated in $[10,11]$ by multiplication of packet size and the average number of corresponding packet type in the buffers of all nodes in the network as shown in (31),(32).

$$
\begin{aligned}
& C D B S=(\mathrm{SCDRFWF}) \quad \times(\text { number of allocated } S C \text { for data }) \quad \times(A H C+1) \\
& =(D S P F \times M A U \div 8) \times\left(N O S-N \times V S P R_{\text {voice }}-N \times V S P R_{\text {video }}\right) \times(A H C+1) \text { Bytes } \\
& \text { calculated }_{\text {video /voice }} \text { Buffer Size }=\left(\text { packet size }_{\text {videolvoice }}\right) \times\left(V P S R_{\text {video/voice }}\right) \times(N) \times(A H C+1) \text { Bytes } \\
& \text { with }\left(V P S R_{\text {video/voice }}\right) \times(N) \times(A H C+1) \text { slots }
\end{aligned}
$$

\subsection{Working principles of the simulation}

The nodes in our system can have any kind of updated information about other nodes in the network. This information is used with novel methods to improve the throughput of the network by decreasing the packet loss ratio given in (33). 
International Journal of Wireless \& Mobile Networks (IJWMN) Vol. 4, No. 2, April 2012

$$
\begin{aligned}
\text { Packet loss ratio } & =\text { lost packets /sent packets } \\
& =\text { lost packets } /(\text { lost packets }+ \text { successfully sent packets })
\end{aligned}
$$

Since the nodes in the network use random way point mobility model [15] with random velocities in the given velocity ranges, it is difficult to predict the complete routes that the packets should follow. So each node recalculates and determines the route and next node of each packet before forwarding it. When congestion and packet losses occur in the network, the transmission rate is decreased using adaptive data transmission method. It is shown in the literature [16] that adaptive rate also has a positive effect on the system throughput. Maximum data rate per user (MDRPU) in a frame is evaluated in $[10,11]$ as given in (34).

$$
\text { MDRPU }=\text { MSU (bytes / s) } \times \frac{T S C F D T}{N O S} \times \frac{1}{N} \times(F L) \times \frac{\text { Succesfully sent } p .}{\text { Succesfully sent } p .+ \text { lost } p .} \text { Bps }
$$

The overall algorithm shown in Fig.2 is also used for evaluating the simulation results of [10, $11]$ in which only the Fastest Path Routing Algorithm is used to decide the routes of the packets. However, in this study, effects of Fastest Path, Ant-Colony, Associativity Based Routing (ABR) and our novel proposed Associativity Based Long life Routing algorithms on novel throughput improvement methods are investigated for different vehicular velocities.

If a node starts to send its packets, it arranges its buffer such that the packets with same next nodes are grouped to be sent together and the packet group whose next node has more free memory will be sent first according to BM method. This process continues during the current OFDMA frame as long as the transmitting node still has packets to send and the frame duration is not over. The role of BM method can be observed in Fig.2.

According to SA algorithm, when a node starts to transmit packets, if the buffer of the freest next node is also full, the transmitter loses its first trial packet of chosen destination and even if the frame duration is not over, it returns its spectrum usage right to the node who gave alert of the 'spectrum need' most. At the end of the frame, the spectrum will again be allocated to another node with most spectral need. The role of SA method is also highlighted in Fig.2.

\subsection{Formulations of packet loss rates and system throughput per user}

\subsubsection{Calculation of the packet loss rates}

In [11], the average packet loss probability at one of the remaining $\mathrm{N}-1$ nodes (excluding the current transmitter and the packets it has) is calculated using (35) - (36). After calculating the probability of loosing a packet in Node ${ }_{\mathrm{n}}$ using (35), the probability of a packet to get lost in any of the nodes can be evaluated as in (36). 
International Journal of Wireless \& Mobile Networks (IJWMN) Vol. 4, No. 2, April 2012

$$
\begin{aligned}
& P_{\text {lost }}(n)= \frac{\frac{\text { Packet Distribution rate of Node } e_{n}}{\text { Sum of Distribution rates of all nodes }} \times \text { Total Packet count }}{\text { Buffer size }} \\
&= \frac{\frac{(n)}{N \times(N+1)} \times \text { Total Packet count }}{2} \text { Buffer size } \\
& P_{\text {loss }}=\frac{1}{N-1} \times P_{\text {lost }}(1)+\frac{1}{N-1} \times P_{\text {lost }}(2)+\ldots+\frac{1}{N-1} \times P_{\text {lost }}(N-1) \\
& P_{\text {loss }}=\frac{1}{(N-1)}\left(\sum_{n=1}^{N-1} P_{\text {lost }}(n)\right)
\end{aligned}
$$

Note that the term $P_{\text {lost }}(n)$ used in (36) - (39) is the one evaluated in (35) and the term $\mathrm{P}_{\text {loss }}$ in (36) - (39) expresses the probability of loosing a packet with used method(s) at any of the nodes. When all methods are activated in our system, the packet loss occurs if and only if all the packets in the buffer of the transmitting node have the next nodes with full buffers. When proposed Buffer Management method is activated, the packet loss rate is calculated as given in (37).

$$
\begin{aligned}
P_{\text {loss_BM } B M} & =\frac{\left(\begin{array}{l}
N-1 \\
1
\end{array}\right) p_{\text {lost }}{ }^{1}+\left(\begin{array}{l}
N-1 \\
2
\end{array}\right) p_{\text {lost }}{ }^{2}+\left(\begin{array}{l}
N-1 \\
3
\end{array}\right) p_{\text {lost }}{ }^{3}+\ldots+\left(\begin{array}{l}
N-1 \\
N-1
\end{array}\right) p_{\text {lost }}{ }^{N-1}}{\sum_{n=1}^{N-1}\left(\begin{array}{l}
N-1 \\
n
\end{array}\right)} \\
P_{\text {loss_BM }}= & \frac{\sum_{n=1}^{N-1}\left\{\left(\begin{array}{l}
N-1 \\
n
\end{array}\right) \times p_{\text {lost }}{ }^{n}\right\}}{\sum_{n=1}^{N-1}\left(\begin{array}{l}
N-1 \\
n
\end{array}\right)}
\end{aligned}
$$

The packet loss rate for the case of applying Spectral Aids method $\left(\mathrm{P}_{\text {loss_SA }}\right)$ without buffer management method (All - Buffer Management in Table 1), is calculated in [11] taking account the buffer state combination of remaining $\mathrm{N}-1$ nodes, by sum of probability of choosing a full buffer and loosing the packet there.

So, for remaining N-1 nodes (excluding the transmitter itself ), we have;

$$
\begin{aligned}
& P_{\text {loss_SA }}=\left(p_{\text {lost }}{ }^{1} \times\left(1-p_{\text {lost }}\right)^{(N-1)-1} \times \frac{1}{N-1}\right)+\left(p_{\text {lost }}{ }^{2} \times\left(1-p_{\text {lost }}\right)^{(N-1)-2} \times \frac{2}{N-1}\right)+\ldots+\left(p_{\text {lost }}{ }^{n} \times\left(1-p_{\text {lost }}\right)^{(N-1)-(n)} \times \frac{n}{N-1}\right) \\
& P_{\text {loss_SA }}=\sum_{n=1}^{N-1} p_{\text {lost }}{ }^{n} \times\left(1-p_{\text {lost }}{ }^{(\mathrm{N}-1)-\mathrm{n})} \times \frac{n}{N-1}\right.
\end{aligned}
$$

When both BM and SA methods are both applied to the system, (39) is evaluated by combining (37) and (38) where confirmations of all evaluated calculation and simulation results are done in [11]. 
International Journal of Wireless \& Mobile Networks (IJWMN) Vol. 4, No. 2, April 2012

$$
P_{\text {loss_rate } A L L}=\sum_{n=1}^{N-1}\left(\frac{\left(\begin{array}{l}
N-1 \\
n
\end{array}\right) \times p_{\text {lost }}{ }^{n}}{\sum_{r=1}^{N-1}\left(\begin{array}{l}
N-1 \\
r
\end{array}\right)} \times\left(1-p_{\text {lost }}\right)^{((N-1)-n)} \times \frac{n}{N-1}\right)
$$

\subsubsection{Throughput calculation taking the bandwidth wastage into account}

The average probabilities of losing packets at any node of determined routes are calculated using (40) and (41) respectively in case of using OFDMA and OFDMCAF [11].

$$
\begin{array}{r}
p_{\text {OFDMA }_{\text {ave }}}=\frac{\frac{\text { Total Packets }}{N}}{\text { Buffer Size }} \\
=\frac{\text { Total Packets }}{N \times \text { Buffer Size }} \\
p_{\text {OFDMCAF }_{\text {ave }}}=\frac{\left(\sum_{n=1}^{N-1}(n) \times \frac{2 \times \text { Total Packets }}{N \times(N+1)}\right)}{(N-1) \times \text { Buffer Size }}
\end{array}
$$

The generalized throughput values for OFDMA and OFDMCAF can also be calculated for different $\mathrm{N}$ values. For this calculation we need; the simulation results only for a fixed $\mathrm{N}$ value, the calculations used in (40) and (41) and set of average hop count values for different $\mathrm{N}$ values evaluated from the routing simulation programs we additionally developed. So, the throughput per user for different $\mathrm{N}$ values with bandwidth wastage can be calculated using (42) and (43) by taking into account the effects of changes on packet loss rates, average hop count, number of nodes, value of $\mathrm{V} \mathrm{PSR}$ video, value of $\mathrm{V} \mathrm{PSR}_{\text {voice }}$ and number of remaining sub-channels for data packets by varying $\mathrm{N}$ value.

$$
\begin{aligned}
& \operatorname{THR}_{\text {with_BW_wastage }}(n)=\frac{\frac{A H C_{N_{\text {sin }}} \text { for N used in Sim. }}{A H C_{n_{\text {ind }}} \text { for } n \text { nodes }} \times[\text { Transmitted packets(bytes) with corresponding packet loss rate }]}{\text { Time }_{\text {sim }}(\mathrm{sec})} B p s \\
& =\frac{\frac{A H C_{N_{\text {sin }}} \text { for } N_{\text {sim. }}}{A H C_{n_{\text {sim }}} \text { forn nodes }} \times\left[\frac{n}{N_{\text {sim }}} \times((\text { sent video })+(\text { sent voice }))+\left((\text { sent }+ \text { lost data }) \times \frac{T S C F D T}{T S C F D T_{\text {sim }}} \times(1-P(n))\right)\right]_{B p s}}{\text { Time }_{\text {sim }}(\mathrm{sec})}
\end{aligned}
$$

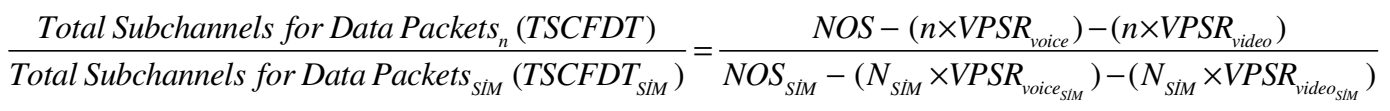

The abbreviation"P" used in (42) is taken as it is calculated in (40) or (41) depending on using it

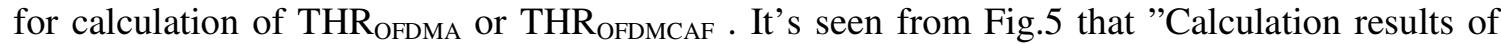
pure OFDMCAF without AR, BM and SA" match with "Simulation results of pure OFDMCAF without AR, BM and SA" and "Calculation results of pure OFDMA without AR, BM and SA" also match with "Unicast analysis results of pure OFDMA without AR, BM and SA" evaluated 
International Journal of Wireless \& Mobile Networks (IJWMN) Vol. 4, No. 2, April 2012

in the literature $[12,13]$. The calculated throughput results for activation cases of each method are also shown in Fig.5, for which all the calculation results match with the corresponding simulation results. Since mentioned works in the literature don't consider the bandwidth wastage on throughput calculation, we have also made the"throughput calculation without bandwidth wastage" to provide the fair comparison on Fig.5. The results in Fig.5 are presented for each number of nodes, with corresponding average hop count, video/voice packet sending rate $\left(\mathrm{VPSR}_{\text {voice }}\right.$ and VPSR video $)$ and other parameter values of the simulation at that instant.

\subsection{Effects of routing algorithms on system throughput}

As it is seen on both (25) and (42) that, the throughput amount is strongly dependent on AHC value such that the throughput is improved by decrease of the AHC value. In addition, according to (31) and (32), each node will need smaller buffers with smaller AHC values. Thus, the routes and hop counts determined by the routing algorithms have very critical role on throughput which has to be investigated. For this purpose, we have developed the routing simulation programs in MATLAB, implementing the Fastest Path routing [17], ANT- Colony routing [18], Associativity based routing (ABR), Alternative Enhanced Associativity based routing (AEABR) [19] and Associatively Tick Averaged Associativity based routing [20] algorithms. At first, effects of Fastest Path and ANT- Colony routing algorithms were taken into comparison for the range of different $\mathrm{N}$ values. The evaluated results are shown in Fig.6 where it is observed that Fastest Path routing algorithm reaches to higher throughput values. Then, Fastest Path routing algorithm and all other novel long life routing algorithms $[19,20]$ are embedded in our simulation system to run simultaneously and make their own decisions for the same conditions. Then, the simulations are run by activating different combinations of novel throughput improvement methods with different vehicular velocities for a fixed $\mathrm{N}$ value $(\mathrm{N}=6)$. Finally, the effects of routing algorithms on system throughput performance with different vehicular velocities are investigated for each of our novel throughput improvement methods. The evaluated results for different vehicular velocities and different routing algorithms are shown in Table 2 and Fig.7.

\section{RESULTS and DISCUSSIONS}

At each simulation run, the improvements made on; efficient bandwidth use, data packet loss rate and throughput of the system are evaluated with and without applying the novel methods for different number of nodes. By not taking one of the methods into progress, we have been able to observe the improvement and the effects of each method on system performance. It is depicted [5] that, the system throughput increase stabilizes when 4 relay nodes are deployed. Thus, before investigating all the results evaluated for a range of all $\mathrm{N}$ values, we focus on an example of results evaluated for totally $\mathrm{N}=6$ nodes including the transmitter and the receiver [11]. Then, the simulation results are confirmed by comparing with other results evaluated in the literature and comparing by the results of calculations we made for packet loss rate and pure system throughput values. For better understanding; Fig.3 and Fig.4 are given to illustrate two examples of our simulation results for application cases of no methods and all methods respectively. 
International Journal of Wireless \& Mobile Networks (IJWMN) Vol. 4, No. 2, April 2012

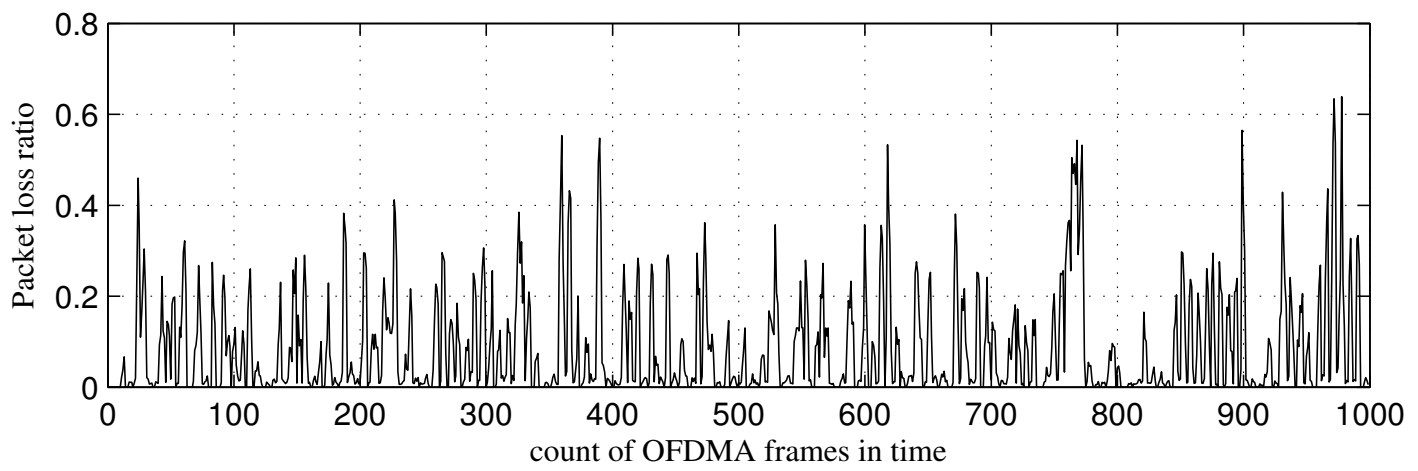

(a)

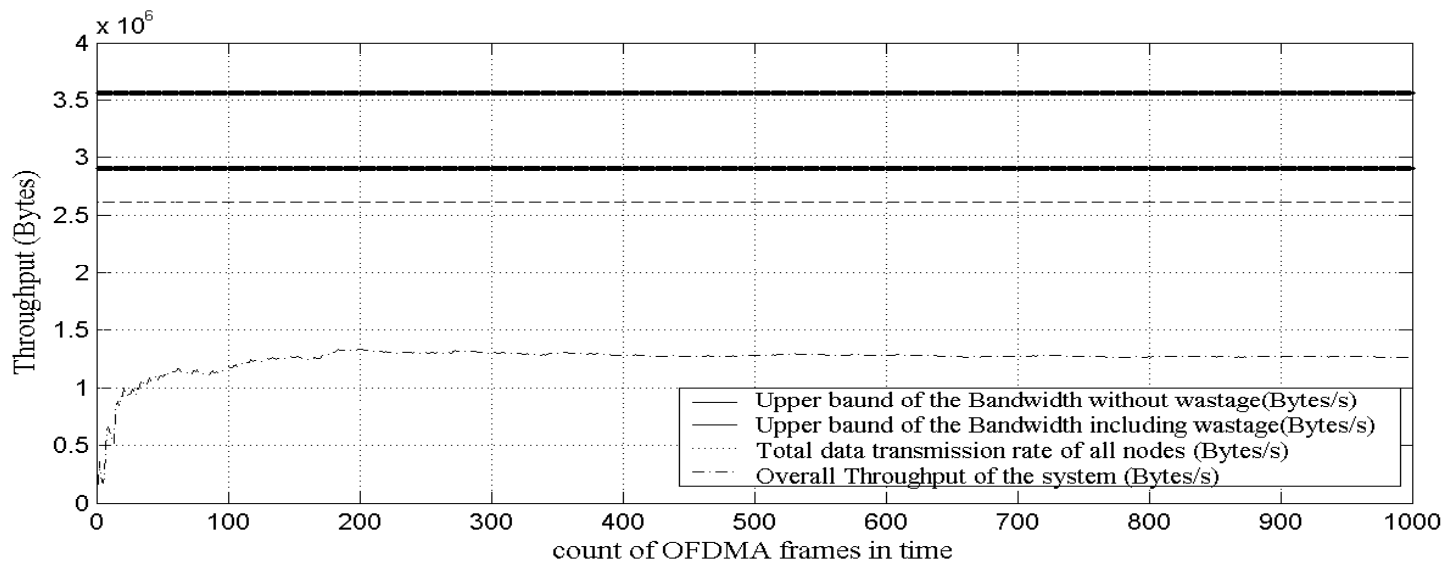

(b)

Fig. 3. (a) Packet loss Ratio of the whole network in last AHC frames period (b) Bandwidth upper bound, data packet generation/ transmission rate and overall throughput of the pure system vs. frame count, with use of half of the calculated data buffer size

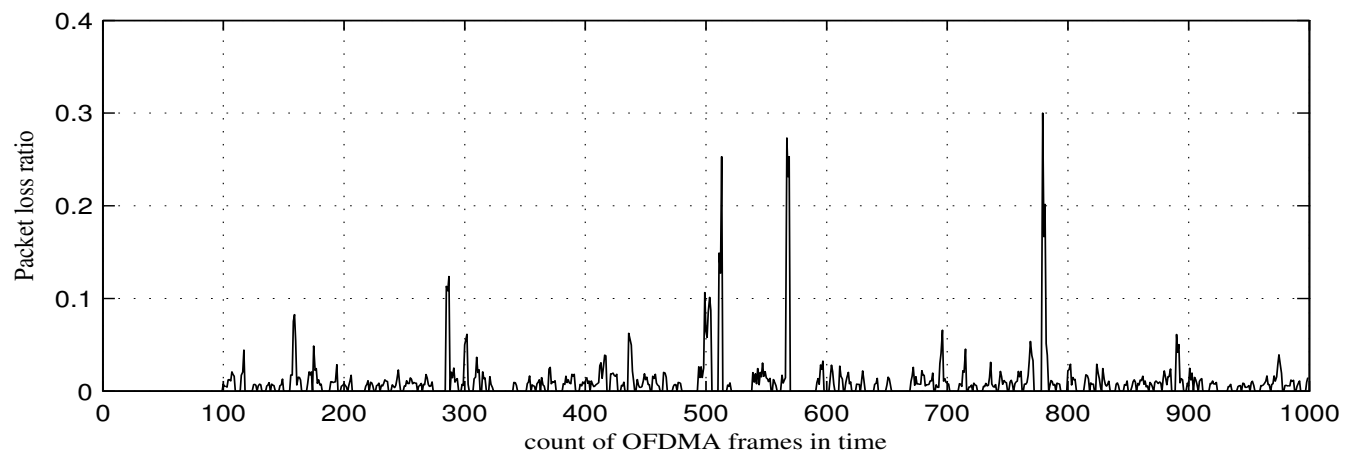

(a) 


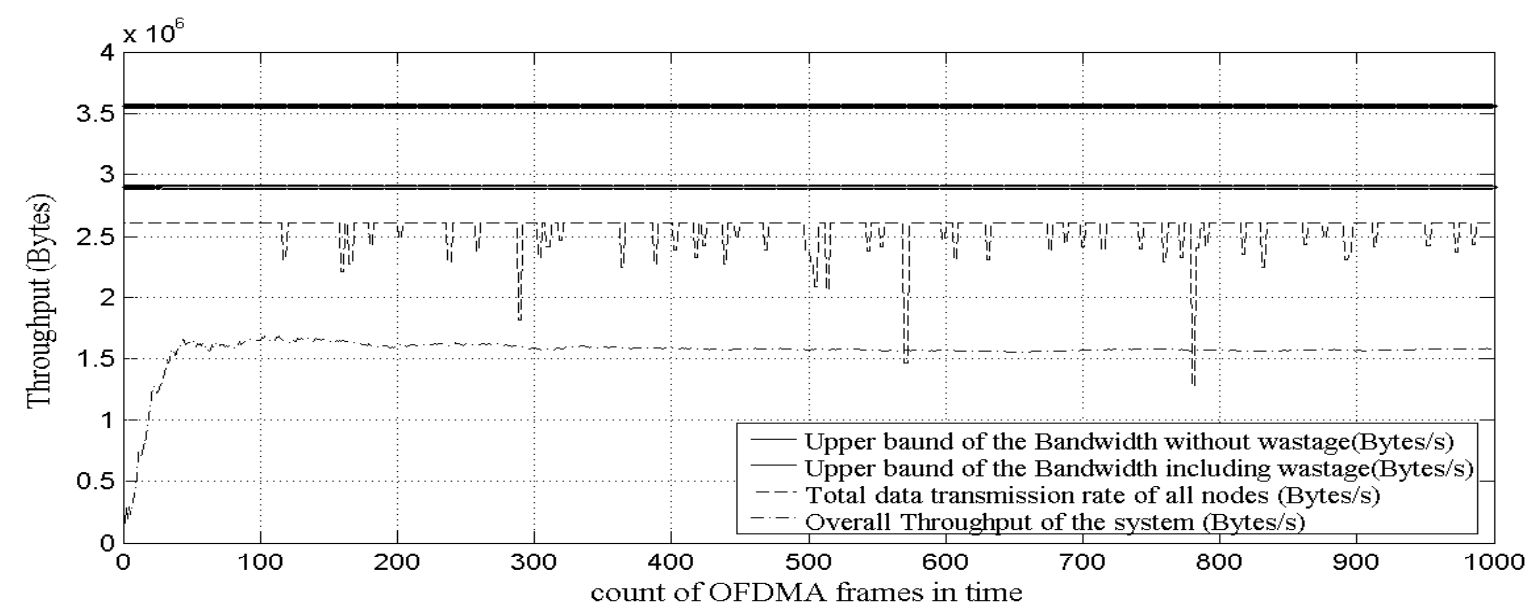

(b)

Fig. 4. (a) Packet loss Ratio of the whole network in last AHC frames period (b) Bandwidth upper bound, data packet generation/ transmission rate and overall throughput of the system vs. frame count, with use of all novel cognitive methods and calculated data buffer size

One example numeric values set of sent/lost packets [11], spectral usage rates and throughput amounts taken from the simulation results for each method are listed in Table 1 (for $\mathrm{N}=6$ ), where the simulation throughput results for a range of different $\mathrm{N}$ values are also available on Fig.5.

Since the system throughput performance is reduced most during the absence case of spectral aids method (see Table 1), it can be said that the greatest throughput improvement is provided by spectral aids method among others. It is also seen that the most efficient spectrum usage is provided when all proposed methods are activated. Increasing the number of nodes also increases the number of total used sub-channels for real time video and voice packets, so, the number of remaining sub-channels to be allocated for data packets decreases.

Thus, the positive effects of our methods on loss ratio of data packets get weaker by increased number of nodes. As a result, it is observed in Fig.5 that, the throughput curves approach each other by increasing $\mathrm{N}$ value. We use 30 sub-channels in our system. There are $\mathrm{N}$ nodes sharing these sub-channels for transmitting their data, voice and video packets. We assume that each node uses, at least one separated sub-channel in one of every 4 frames [7] (with VPSR $_{\text {video }}>=$ 1/4) for its own video conversations and at least one separated sub-channel in every frame [7] (with $\operatorname{VPSR}_{\text {voice }}>=1$ ) for its own voice conversations.

Thus, [NOS $\left.\left.-\left(N \times V P S R_{\text {voice }}+N \times V P S R_{\text {video }}\right)\right)\right]$ sub channels will be used by $\mathrm{N}$ nodes for data packets, so we have $[N O S-(N+(N / 4))]>=0$ and $N<=24$ for $N O S=30$. That's why the value of $\mathrm{N}$ is increased up to 24 in our simulation (see Fig. 5). 
International Journal of Wireless \& Mobile Networks (IJWMN) Vol. 4, No. 2, April 2012

Table 1. The simulation Results for different methods

\begin{tabular}{|c|c|c|c|c|c|c|c|c|c|c|c|}
\hline \multirow{2}{*}{$\begin{array}{c}\text { Simulation } \\
\text { Output } \\
\text { Data in } 5 \\
\text { seconds } \\
\text { (for } \mathrm{N}=6 \text { ) }\end{array}$} & \multicolumn{2}{|l|}{ 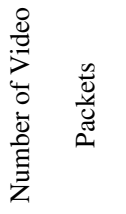 } & \multicolumn{2}{|c|}{ 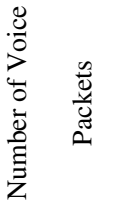 } & \multicolumn{2}{|c|}{ 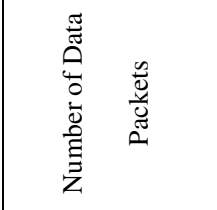 } & \multicolumn{2}{|c|}{ 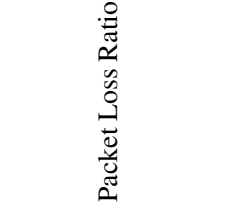 } & \multirow{2}{*}{ 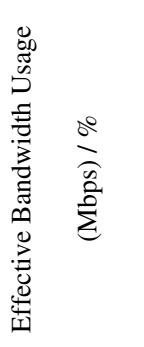 } & \multirow{2}{*}{ 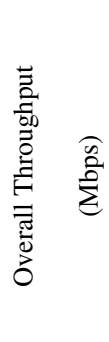 } & \multirow{2}{*}{ 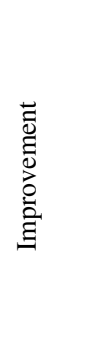 } \\
\hline & $\begin{array}{l}\overline{\bar{\nu}} \\
\text { ñ }\end{array}$ & 㟥 & 节 & 菺 & 离 & 蒿 & $\begin{array}{l}\text { Video/ } \\
\text { Voice }\end{array}$ & 泀 & & & \\
\hline No methods & 494 & 0 & 2389 & 0 & 29344 & 4456 & $0 \%$ & $13.18 \%$ & $2.64 / 90$ & 1.22 & $0 \%$ \\
\hline All - adaptive & 507 & 0 & 2576 & 0 & 35953 & 653 & $0 \%$ & $1.78 \%$ & $2.79 / 95$ & 1.48 & $22 \%$ \\
\hline All + larger & 515 & 0 & 2532 & 0 & 36691 & 58 & $0 \%$ & $0.16 \%$ & $2.80 / 96$ & 1.51 & $24 \%$ \\
\hline All - buffer & 517 & 0 & 2582 & 0 & 33978 & 654 & $0 \%$ & $1.89 \%$ & $2.75 / 94$ & 1.41 & $15 \%$ \\
\hline All - spectral & 502 & 0 & 2575 & 0 & 31419 & 786 & $0 \%$ & $2.44 \%$ & $2.78 / 95$ & 1.30 & $7 \%$ \\
\hline All + Calc. B & 515 & 0 & 2544 & 0 & 37631 & 182 & $0 \%$ & $0.48 \%$ & $2.80 / 96$ & 1.55 & $27 \%$ \\
\hline
\end{tabular}

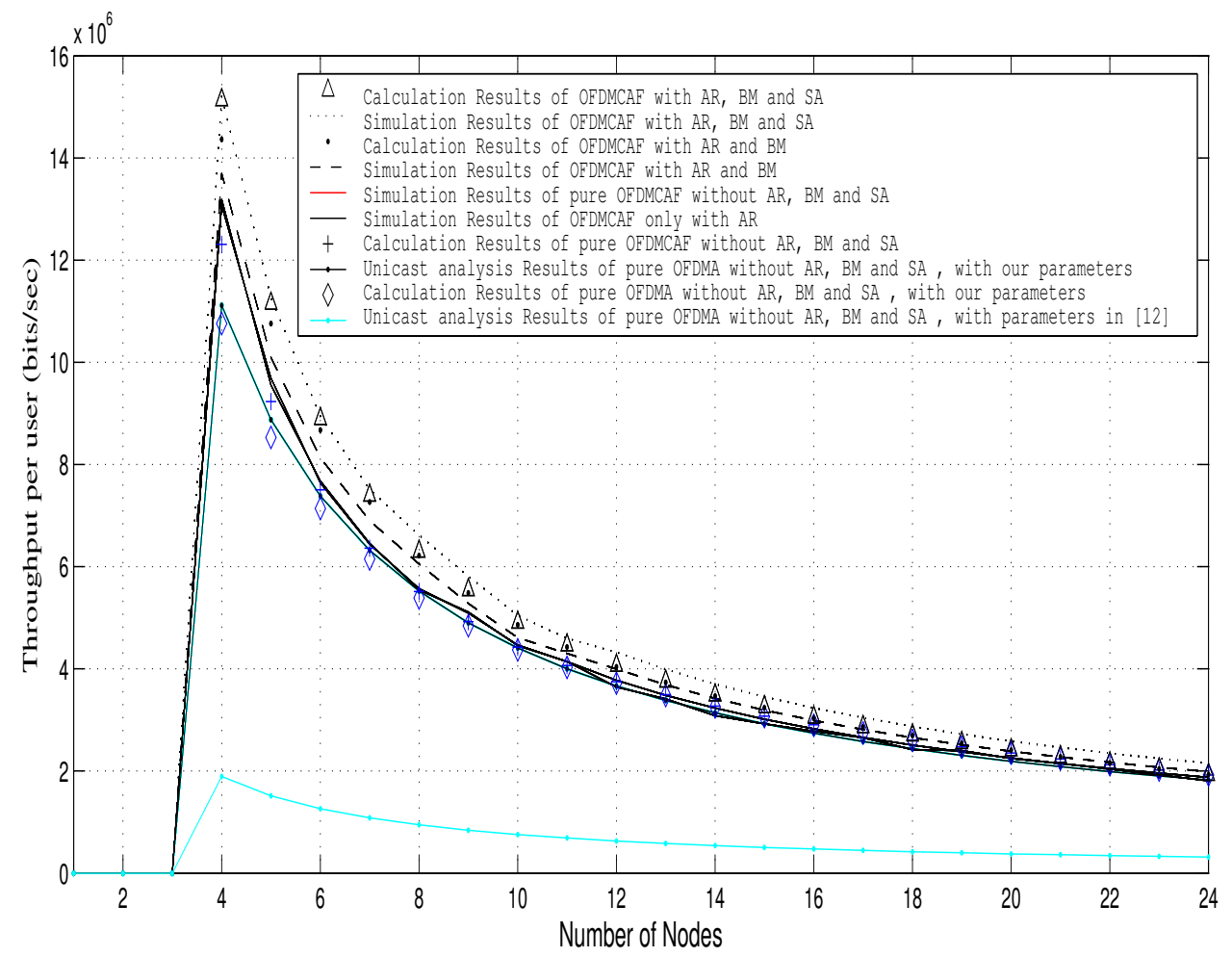

Fig. 5. Throughput results of OFDMA, OFDMCAF and unicast asymptotic analysis [12, 13] using fastest path routing algorithm 
Before investigating the effects of all routing algorithms on the system throughput improvement methods, as the final purpose of this work, we investigated the effects of only the Fastest Path and ANT-Colony routing algorithms on throughput improvement of our methods with various numbers of nodes. It is shown in Fig. 6 that the novel methods provide throughput improvement for both routing algorithms. But, it is seen that the system always provides higher throughput for the pure system with Fastest Path routing algorithm.

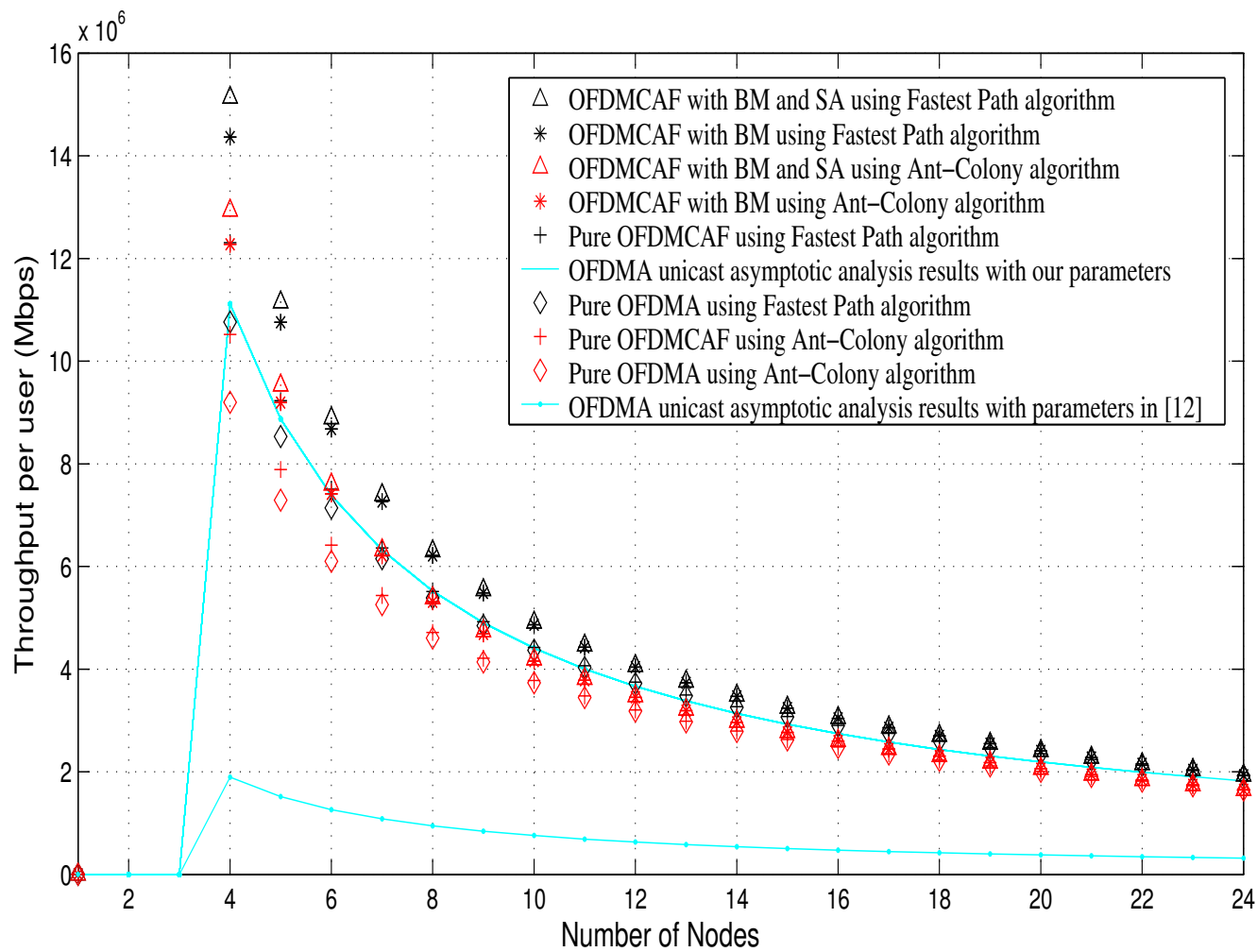

Fig. 6. The throughput performances of Ant-Colony and Fastest path routing algorithms

Finally, effects of Fastest Path routing algorithm (which is shown to always provide better performance than Ant Colony Routing algorithm) and Long Life routing algorithms (ABR, AEABR and ATAABR) on each novel proposed throughput improvement method are investigated in terms of instant and average; hop counts and system throughput value for different vehicular velocities in a mobile 802.16j network.

By running the simulator and processing all routing algorithms simultaneously for absolutely same conditions, the generated simulation results of all methods for different vehicular velocities are illustrated in Table 2. It is seen from the simulation results in Table 2 and Fig.7 for different vehicular velocity ranges that; the throughput is improved by ABR more than improved by Fastest Path routing algorithm (which is shown to provide greater throughput improvement than Ant-Colony routing algorithm in Fig.6). On the other hand the throughput is improved more by ATAABR then ABR where the throughput is improved most by our novel AEABR algorithm for all vehicular velocities. It is observed that, higher node velocities cause the throughput to decrease but the throughput amount decreases most for the vehicular velocities greater than 60 $\mathrm{km} / \mathrm{h}$. 
International Journal of Wireless \& Mobile Networks (IJWMN) Vol. 4, No. 2, April 2012

Table 2. Average hop counts and throughput values of routing Algorithms for vehicular velocities between $25-40 \mathrm{~km} / \mathrm{h}, 60-80 \mathrm{~km} / \mathrm{h}$ and $100-110 \mathrm{~km} / \mathrm{h}$

\begin{tabular}{|c|c|c|c|c|c|c|c|c|c|c|c|c|c|}
\hline \multicolumn{2}{|c|}{ Routing Method } & \multicolumn{4}{|c|}{$\begin{array}{l}25-40 \mathrm{~km} / \mathrm{h} \text { vehicular } \\
\text { velocity }\end{array}$} & \multicolumn{4}{|c|}{$60-80 \mathrm{~km} / \mathrm{h}$ vehicular velocity } & \multicolumn{4}{|c|}{$\begin{array}{c}100-110 \mathrm{~km} / \mathrm{h} \text { vehicular } \\
\text { velocity }\end{array}$} \\
\hline \multirow{3}{*}{$\begin{array}{c}\text { Fastest } \\
\text { Path } \\
\text { Algorithm }\end{array}$} & \multirow{3}{*}{$\begin{array}{c}\text { Hop Count } \\
\text { Throughput } \\
\text { (Mbps) }\end{array}$} & \multicolumn{4}{|c|}{3.16} & \multicolumn{4}{|c|}{3.16} & \multicolumn{4}{|c|}{3.16} \\
\hline & & NO & $\mathrm{BM}$ & SA & BM,SA & $\mathrm{NO}$ & $\mathrm{BM}$ & SA & BM,SA & $\mathrm{NO}$ & BM & SA & $\mathrm{BM}, \mathrm{SA}$ \\
\hline & & 1.22 & 1.30 & 1.32 & 1.33 & 1.22 & 1.30 & 1.32 & 1.33 & 1.22 & 1.30 & 1.32 & 1.33 \\
\hline $\mathrm{ABR}$ & Hop Count & \multicolumn{4}{|c|}{2.12} & \multicolumn{4}{|c|}{2.16} & \multicolumn{4}{|c|}{2.20} \\
\hline \multirow{2}{*}{ Algorithm } & \multirow{2}{*}{$\begin{array}{c}\text { Throughput } \\
\text { (Mbps) }\end{array}$} & $\mathrm{NO}$ & BM & SA & $\mathrm{BM}, \mathrm{SA}$ & NO & $\mathrm{BM}$ & SA & $\mathrm{BM}, \mathrm{SA}$ & $\mathrm{NO}$ & BM & SA & BM,SA \\
\hline & & 1.82 & 1.94 & 1.97 & 1.98 & 1.79 & 1.90 & 1.93 & 1.95 & 1.75 & 1.87 & 1.90 & 1.91 \\
\hline ATAABR & Hop Count & \multicolumn{4}{|c|}{2.10} & \multicolumn{4}{|c|}{2.20} & \multicolumn{4}{|c|}{2.13} \\
\hline \multirow[t]{2}{*}{ Algorithm } & \multirow{2}{*}{$\begin{array}{c}\text { Throughput } \\
\text { (Mbps) }\end{array}$} & $\mathrm{NO}$ & BM & SA & $\mathrm{BM}, \mathrm{SA}$ & NO & $\mathrm{BM}$ & SA & $\mathrm{BM}, \mathrm{SA}$ & $\mathrm{NO}$ & $\mathrm{BM}$ & SA & $\mathrm{BM}, \mathrm{SA}$ \\
\hline & & 1.84 & 1.96 & 1.99 & 2.00 & 1.75 & 1.87 & 1.90 & 1.91 & 1.81 & 1.93 & 1.96 & 1.97 \\
\hline AEABR & Hop Count & \multicolumn{4}{|c|}{2.02} & \multicolumn{4}{|c|}{2.09} & \multicolumn{4}{|c|}{2.08} \\
\hline \multirow[t]{2}{*}{ Algorithm } & \multirow{2}{*}{$\begin{array}{c}\text { Throughput } \\
\text { (Mbps) }\end{array}$} & NO & BM & SA & $\mathrm{BM}, \mathrm{SA}$ & NO & $\mathrm{BM}$ & SA & $\mathrm{BM}, \mathrm{SA}$ & $\mathrm{NO}$ & $\mathrm{BM}$ & SA & $\mathrm{BM}, \mathrm{SA}$ \\
\hline & & 1.91 & 2.03 & 2.07 & 2.08 & 1.85 & 1.96 & 2.00 & 2.01 & 1.85 & 1.97 & 2.01 & 2.02 \\
\hline
\end{tabular}

$x 10^{6}$

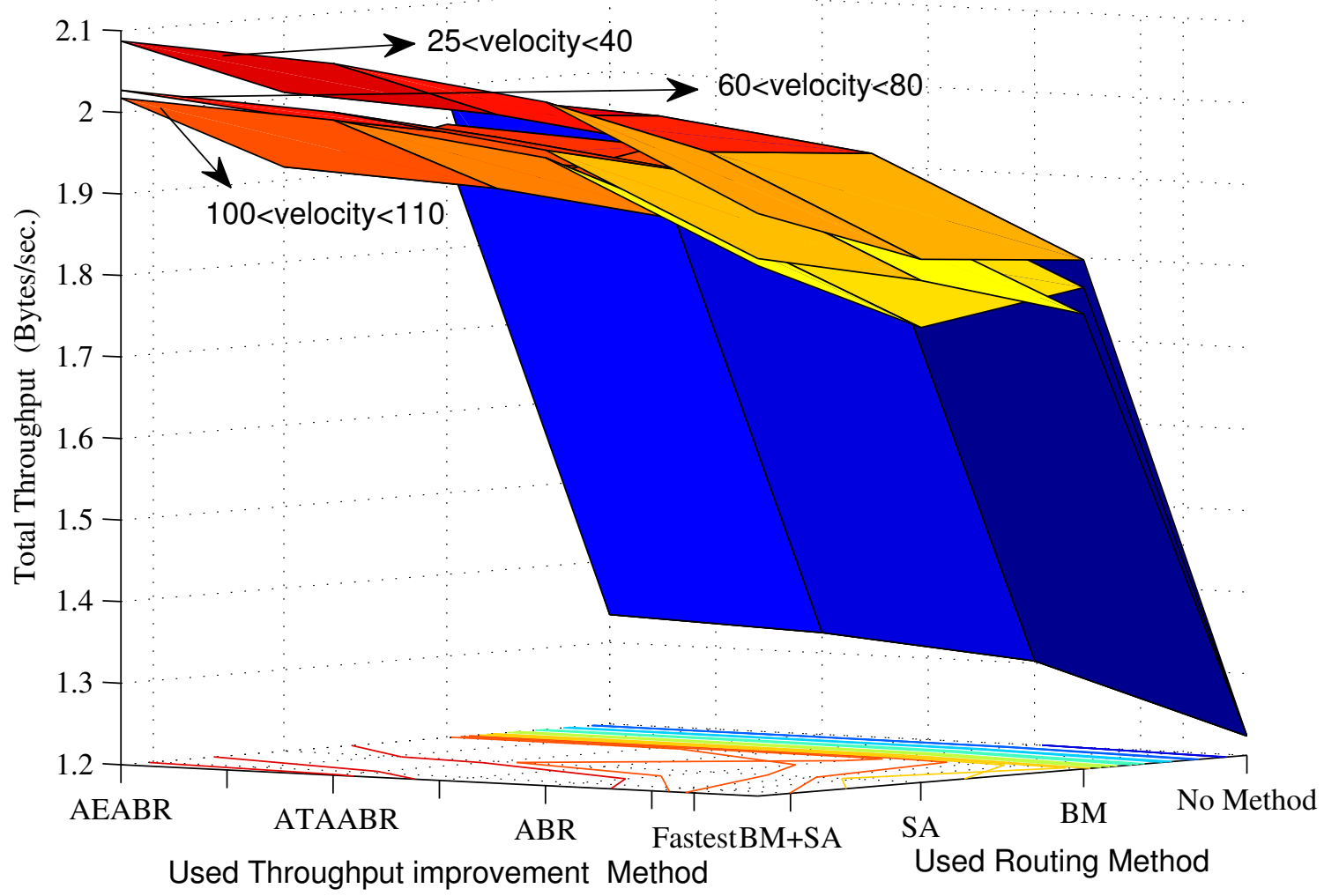

Fig. 7. The throughput improvement by using different; throughput improvement methods vs. routing methods vs. vehicular velocities.

It is shown in Fig.7 that the novel proposed throughput improvement algorithms (BM, SA, $\mathrm{BM}+\mathrm{SA}$ ) are succeeded in improving the system throughput for Fastest Path, Ant-Colony, ABR and other novel Associativity Based Routing algorithms (ATAABR and AEABR). 
International Journal of Wireless \& Mobile Networks (IJWMN) Vol. 4, No. 2, April 2012

\section{CONCLUSION}

To the best of our knowledge, this is the first analytically confirmed event-driven simulative work that investigates the effects of novel routing algorithms on each throughput improvement method for different velocities.

In this work, it is illustrated that the known routing algorithms (ABR, Fastest Path and Ant Colony) and our novel proposed Associativity Based Routing algorithms (AEABR [19] and ATAABR [20]) which generate routes with longer life times, all provide throughput improvement when they are used with the novel OFDMCAF method.

It is shown in this work that, the Fastest Path routing algorithm improves the throughput always better than the Ant Colony routing algorithm. On the other hand all Associativity Based Routing algorithms provide better system throughput performance than others, where proposed AEABR algorithm provides the best performance for all throughput improvement methods and with all vehicular velocities. In case of using no throughput improvement method ABR provides $49 \%$ throughput improvement, ATAABR provides $51 \%$ throughput improvement, AEABR provides $56 \%$ throughput improvement with respect to the Fastest Path algorithm for the vehicular velocities up to $60 \mathrm{~km} / \mathrm{h}$ and the throughput improvement effect decreases by increased vehicular velocity.

It must be noted that, the results of the methods that we propose can also be evaluated using any other popular event-driven network simulator in the area. The methods, algorithms, formulations and results of this work can be used in designing or analyzing a unicast mobile multimedia network considering the routing algorithms decreasing packet loss rate and improving the throughput.

\section{REFERENCES}

[1] Mitola, III J. \& Maguire, G, (1999) "Cognitive radio : making software radios more personal", IEEE personal communications,. 13-18.

[2] Mitola, III J. (2000) "Cognitive radio and integrated agent architecture for software defined radio", Dissertation KTH Royal institute of technology.

[3] Hu, X. \& Li, B. (2010) "Efficient resource allocation with flexible channel cooperation in OFDMA cognitive radio networks". INFOCOM, 1-9.

[4] Sirinivasa, S. \& Jafar, A. (2006) "The throghput potantial of cognitice radio : A theoretical perspective", ACSSC 2006. 221-225.

[5] Genca, V. \& Murphy, S. (2008) "Performance analysis of transparent relays in 802.16j MMR networks". WIOPT 2008. 273-281.

[6] Salameh, H. B. \& Krunz, M. (2009) "Spectrum sharing with adaptive power management for throughput enhancement in dynamic access networks." Tech. Rep. TR-UA-ECE-2009-. University of Arizona, Department of ECE.

[7] Kumar, A. (2008), "Mobile broadcasting with WIMAX”, Elsevier Inc.

[8] WIMAX FORUM, (2006) "Mobile WIMAX - part 2:a comperative analysis"

[9] WIMAX FORUM, (2006) "Mobile wimax - part 1:a technical overview and performance evaluation." 
[10] Preveze, B. \& Safak, A. (2010) "Throughput maximization of different signal shapes working on 802.16e mobile multi-hop network using novel cognitive methods." Recent trends in wireless and mobile networks, WIMO 2010 Turkey, 2010. 71-86.

[11] Preveze, B. \& Safak, A. (2010), "Throughput improvement of mobile multi-hop wireless networks." International journal of wireless and mobile networks, Vol. 2 No.3 120-140.

[12] Girici, T. (2009) "Asymptotic throughput analysis of multicast transmission schemes", International journal of electronics and communications (AEU) Vol. 63 901-905.

[13] Song, G. \& Li, Y. (2006) "Asymptotic throughput analysis of channelaware scheduling". IEEE transactions on communications 54-10, 1827-1834.

[14] Mach, P. \& Bestak, R. (2008), "WIMAX throughput evaluation of conventional relaying", Telecommunication systems Journal Vol. 38 No. 1-2, 11-17.

[15] Deborah, E., Daniel, Z, Li, T. , Yakov R. \& Kannan, V. (1995), "Source demand routing packet format and forwarding specification" Mobi Com 1995. 1-7.

[16] Iannone, L. \& Fdida, S. (2006), "Can multi rate radios reduce end to end delay in mesh network? a simulation case study", MobiHoc 2006. 15-22.

[17] Yi, X. \& Wanye, W. (2008), Finding the fastest path in wireless networks. IEEE ICC 2008. 31883192.

[18] Sivajothi, M. \& Naganathan, E. N. (2008), "An ant-colony based routing protocol to support multimedia communication in ad-hoc wireless networks", International journal of computer science and network security Vol. 8 No.7, 21-28.

[19] Preveze, B. \& Safak, A. (2009), "Alternative Enhancement of Associatively Based Routing (AEABR)for mobile networks. MONAMI 2009. 67-77.

[20] .Preveze, B. \& Safak, A. (2009) "Associativity Tick Averaged Associativity Based Routing (ATAABR) for real time mobile networks". ELECO 2009.

\section{Author}

Barbaros Preveze received the B.S. and M.S. degrees from Çankaya University Department of Computer Engineering in Ankara, Turkey, in 2001 and 2004 respectively. He received the Ph.D. degree from Başkent University Department of Electrical and Electronics Engineering in Ankara Turkey (2011). He is now working as an Assist. Prof. Dr. at Çankaya University Department of Electronics and Communications Engineering. His research interests are in computer communications, wireless communications and networking, with a current focus on throughput improvement and routing of multi-hop wireless networks.

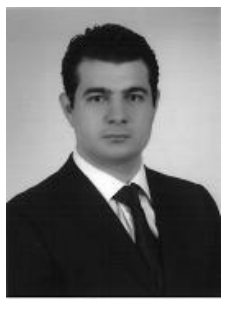

